\title{
COMENTARIOS SOBRE CNEMEPLATIINI JACQUELIN DU VAL, 1861 (COLEOPTERA, TENEBRIONIDAE) DE LA PENÍNSULA IBÉRICA E ISLAS CANARIAS
}

\author{
J. Ferrer' ${ }^{1}$ A. Castro Tovar² \& J. L. Lencina Gutiérrez ${ }^{3}$
}

\begin{abstract}
RESUMEN
La composición taxonómica y distribución geográfica de los representantes de la tribu Cnemeplatiini en la Península Ibérica y Canarias, se establece tras el estudio de los tipos disponibles. Se corrigen varios errores de trabajos recientes. Cnemeplatia laticeps (Wollaston, 1857) descrita de Madeira, se cita como nuevo para la isla de La Palma, Islas Canarias. Cnemeplatia atropos Costa, 1847 es tratado como una especie válida. Cnemeplatia rufa Tournier, 1874 stat. rest. y C. mouchampsi Español 1948 stat. nov. son consideradas como especies válidas, no como subespecies geográficas de Cnemeplatia atropos Costa, 1847, en base a su morfología. El nombre Cnemeplatia rufa Tournier, 1874 tiene prioridad sobre C. atropos africana Kaszab, 1938 syn. nov. Se presentan figuras de los caracteres morfológicos discriminatorios para identificar las diferentes especies descritas de Europa y NO de África.

Palabras clave: Peninsula Ibérica, NO de África, Coleoptera, Tenebrionidae, Cnemeplatiini, Cnemeplatia, Lepidonecmeplatia, Philhammus, biogeografía.
\end{abstract}

\begin{abstract}
Comments on Cnemeplatiini Jacquelin du Val, 1861 (Coleoptera, Tenebrionidae) of the Iberian Peninsula and Canary Islands

The taxonomy and geographic distribution of the Iberian and Canary Islands representatives of the tribe Cnemeplatiini, is established, after study of available types. Several errors in recent papers are corrected. Cnemeplatia laticeps (Wollaston, 1857) described from Madeira, is cited as new record for the isla of La Palma, Canary Islands. Cnemeplatia atropos Costa 1847 is a valid species. Cnemeplatia rufa Tournier, 1874 stat. rest. and C. mouchampsi Español 1948 stat. nov. are considered as valid species and not geographical subspecies of Cnemeplatia atropos Costa, 1847, based on morphology. The name Cnemeplatia rufa Tournier, 1874 has priority over C. atropos africana Kaszab, 1938 syn. nov. Figures of discriminatory characters to identify all European and Northwestern African described species are given.
\end{abstract}

Key words: Iberian Peninsule, Norwestern Africa, Coleoptera, Tenebrionidae, Cnemeplatiini, Cnemeplatia, Lepidonecmeplatia, Philhammus, biogeography.

Department of Entomology, Swedish Museum of Natural History, S-10405 Stockholm, Suecia.

Muñoz Garnica 10, $2^{\circ}$ izq., 23001 Jaén, España.

Departamento de Zoología y Antropología Física. Área de Biología Animal, Facultad de Veterinaria, Universidad de Murcia, Campus de Espinardo, Ado. 4021, 30071, Murcia, España. 


\section{Introducción}

Los géneros Cnemeplatia Costa, 1847 y Philhammus Fairmaire, 1870 fueron transferidos de la tribu Melanimini Seidlitz, 1894 a la tribu Cnemeplatiini Jacquelin du Val, 1861, por Csiki (1953) y, puesto que presentan el edeago en posición dorsal, han sido transferidos a la subfamilia Pimeliinae (Bouchard et al., 2005; Löbl \& Smetana, 2008). El género Cnemeplatia ha sido objeto de una revisión por Kaszab (1938). Esta trabajo fue completado con la descripción (en base a un único ejemplar) de Cnemeplatia atropos mouchampsi Español, 1948, del Congo. Más tarde, Español (1959) redescribe la subespecie C. atropos africana y traduce la diagnosis de Kaszab (1938), pero remite al lector a una figura idéntica a la que representa a $C$. a. atropos según Kaszab (1938), que se caracteriza por presentar los lados del pronoto sinuados en el tercio basal, netamente salientes hacia afuera y por presentar las fosetas del pronoto bien marcadas. Más tarde, Español (1962) se ocupa de nuevo del género Cnemeplatia, estudiando los Opatrinae de Canarias. En este trabajo menciona a Cnemeplatia laticeps (Wollaston, 1857), pero le atribuye ojos cónicos, un carácter que el tipo no presenta, siendo redondos y salientes, y que según la descripción de Kaszab (1938), es propio de Cnemeplatia theryi, aunque éste carácter está muy exagerado en la figura que reproduce Kaszab (fig. 21 cf. 22).

Estos problemas dificultan la identificación de las especies ibéricas del género, que sigue presentando dificultades (Lencina Gutiérrez et al., 2007); además la subespecie $C$. atropos africana no se incluye en la Fauna Europea (Fattorini, 2005). Otro problema relacionado con la identificación de Cnemeplatiini es el carácter cosmopolita de algunas especies. Por ejemplo en 1984 aparece en Chile Lepidocnemeplatia sericea (Horn, 1870), a su vez aclimatada en USA procedente de México, dando lugar a medidas aduaneras en Chile para rechazar la importación de espárragos de USA.

Estos problemas y errores nos llevaron a revisar el conjunto de estos trabajos y realizar el estudio comparado de los tipos disponibles, de forma se pueda identificar correctamente el material previamente estudiado por los diversos autores y el nuevo material.

Esta revisión, basada en el estudio del material típico, propone la sinonimia, de Cnemeplatia atropos africana Kaszab (1938) syn. nov. con Cnemeplatia rufa Tournier (1874) stat. rest.

Se establece que $C$. atropos y $C$. rufa son dos especies simpátricas que cohabitan, tanto en la
Península Ibérica como en el Norte de África, en Argelia, no dos vicariantes geográficos de una misma especie. Las diferencias morfológicas constatadas en el habitus y en el edeago de C. atropos mouchampsi Español (1948) de la república del Congo, son tan evidentes que consideramos adecuado su tratamiento específico: C. mouchampsi Español (1948) stat. nov.

\section{Material y método}

Esta revisión se basa en el estudio del material europeo y africano de la colección Wollaston, conservado en The Natural History Museum (NHM), Londres, del Muséum national d'Histoire naturelle, (MNHN), Paris, del Termeszettudomanyi Muzéum, $(\mathrm{HMNH})$, Budapest, en la coleccion de G. Frey, determinada por Carlo Koch, conservada en el Naturhistorisches Museum, (NHMB), Basilea que contiene así mismo sintipos de Wollaston; los ejemplares de Cnemeplatia determinados por F. Español, conservados en el Museo Nacional de Ciencias Naturales (MNCN), Madrid, los materiales del Naturhistoriska riksmuseet (NRM), de Estocolmo, los ejemplares determinados y enviados en la década de 1970, a JF por Francisco Español y por Zoltán Kaszab, conservados en la colección JF y los ejemplares determinados por F. Español del Museo de Ciencias Naturales de Barcelona (MCNB) y de la Estación Experimental de Zonas Àridas (EEZA), Almería. Estos materiales se completaron con material procedente de capturas recientes, identificado por Lencina et al. (2007), como C. atropos, de las provincias de Murcia y de Cádiz, y realizando JLL y ACT intensos muestreos para localizar Cnemeplatia en diversos puntos geográficos.

Para determinar la correspondencia entre las descripciones originales y la iconografía respectiva se han estudiado las proporciones de los caractéres a partir de las fotografías previas del material tipo estudiado.

\section{Resultados y discusión}

Viñolas y Cartagena (2005) atribuyen la tribu Cnemeplatiini a Csiki (1953) y no a Jacquelin du Val (1861) y confunden los géneros Cnemeplatia Costa, 1847 y Lepidocnemeplatia Kaszab, 1938, separados por Kaszab (1966). Estos autores mezclan así mismo, la respectiva distribución geográfica de estos géneros: en efecto, limitan erróneamente la distribución de Cnemeplatiini (p. 240) en el con- 
tinente africano, a la mitad septentrional de África, ignorando los taxones Cnemeplatia angusta Kaszab, 1938 descrita de Tanzania y C. atropos mouchampsi Español, 1948, descrita del Congo. Luego, citan (p. 241) el género Cnemeplatia de todo el Sur de Europa, de Madeira, de la India, cayendo en contradicción con la distribución que previamente dan de la tribu, del África tropical. El género Cnemeplatia aparece así mismo en Uzbekistán (Karakalpakskoy, República de Karakal), de donde recientemente Medvedev (1982), describió C. oxyana, y C. zarudni Medveded, 1991 de la India, aparte de estas omisiones, extienden erróneamente la representación de Cnemeplatia a "California y Panamá", sin embargo, todos los taxones neotropicales pertenecen al género Lepidocnemeplatia Kaszab (1938), apareciendo éstos también en México: Lepidonecmeplatia laticollis (Champion, 1884) así como en Chile y Argentina (Viana, 1960). En cuanto a la presencia del género en California, es debida a una importación de la especie originaria de Panamá L. laticollis, sinónima de Cnemeplatia sericea Horn, 1870 (Kaszab, 1938; Triplehorn, 1987; Ferrer \& Odegaard, 2005).

Los géneros Philhammus, Cnemeplatia y Lepidocnemeplatia pueden ser separados con las claves de Kaszab (1938, 1966).

Philhammus Fairmaire, 1870

ESPECIE TIPO: Philhammus sericans Fairmaire, 1870

El género Philhammus Fairmaire, 1870, y su representación en África sub-equatorial y meridional ha sido objeto de diversos estudios recientes: Ardoin (1976), Ferrer (1995) y Medvedev (2005), que han descrito nuevas especies. Philhammus presenta una vasta distribución ibero-magrebí (Serrano et al. 2003), norteafricana, sahélica, tropical y subtropical, extendiéndose por el Centro de África, 1legando a Namibia y Botswana.

El género Philhammus Fairmaire, 1870 se encuentra representado en la Península ibérica por una especie ibero-magrebí, Philhammus sericans Fairmaire, 1870 (Cárdenas et al., 2004).

Philhammus sericans Fairmaire, 1870

Material eXAminado: Huelva: Parque Nacional de Doñana, Cerro de los Ànsares, 27.IX.2000, M.M. Cárdenas leg. (CJF); Cádiz, J.L. Tórres Méndez, CJF.

Talla: $3 \mathrm{~mm}$. Cuerpo subparalelo, oblongo, convexo, cubierto de una pubescencia sedosa, de un tono pajizo, clara, los ojos poco visibles dorsalmente, tan anchos como a lo sumo, dos artejos antenales, con antenas proporcionalmente cortas, los tres apicales en maza, pronoto un poco más ancho transversalmente, casi cuadrado, los lados rectos, los ángulos anteriores y posteriores obtusos, con un sillón superficial en el medio; élitros oblongos, atenuados obtusamente hacia el ápice, con estrías leves, de puntos superficiales. Protibias cortas, triangulares y muy anchas, con un lóbulo obtuso, armado de espolones en su parte distal. El interno, más fuerte y más largo, tan largo como el protarso, mesotibias con un espolón interno muy largo, las metatibias con un espolón tan largo al menos, como el primer artejo metatarsal.

La presencia de $P$. sericans (Fig. 25) en la Península Ibérica fue indicada por Cárdenas et al. (2004), colectada en el Coto Doñana, y omitida por Viñolas y Cartagena, (2005) (Ferrer, 2006). Esta especie existe así mismo en Cádiz, de donde hemos visto ejemplares colectados por J. L. Torres Méndez (CJF), indicación de su presencia estable en la Península.

\section{Cnemeplatia Costa, 1847}

ESPECIE TIPO: Cnemeplatia atropos Costa, 1847

Cnemeplatia atropos Costa, 1847

Autocera anticipes Wollaston, 1857: 155

Material eXAminado: La colección de A. Costa se conserva en el Museo de Nápoles, por ello consideramos los ejemplares determinados por Aliquó et al. (2007), de Nápoles como ejemplares cum tipo comp.; así como los ejemplares de Nápoles del Museo de Basel (NHMB): Italia: Nápoles, (10) NHMB; Francia: Córcega: Ajaccio, (NHMB); Grecia: Corfú, Paganetti (NRM, NHMB); Corfú, Laguna, A. Schuster det. (NRM, NHMB); Attica, (NHMB); Monte Parnaso: Parnass, Krüper leg. Coll. Stöklein (NHMB); Hungría: coll. Rey (CCEC, Lyon); España: Palencia (Paganetti) (NRM y NHMB); Turquía: Side, Antalya región (CJF); Argelia: Orán (NHMB). En el MNCN, Madrid existe así mismo un ejemplar etiquetado 360 , sin otros datos, pero que probablemente procede de Argelia, pues un ejemplar de la colección Pérez Arcas, pertenenciente a $C$. rufa de Orán, lleva el número 364 de la misma serie $(\mathrm{MNCN})$.

El material examinado de la Península Ibérica procede de: Murcia: Jumilla, La Alquería, 7.III.2004, 30.XI.2003, J.L. Lencina leg. (suelo de olivo); Valencia: Fuente La Higuera, 12.V.1990, J.L. Lencina leg.(CJLL).

Siendo relativamente variables, los ejemplares de C. atropos de Italia y de Grecia, así como de Argelia y de Turquía presentan las fosetas del pronoto netas, a veces muy indicadas y los élitros largos, correspondiendo casi exactamente a la figura 5 publicada por 

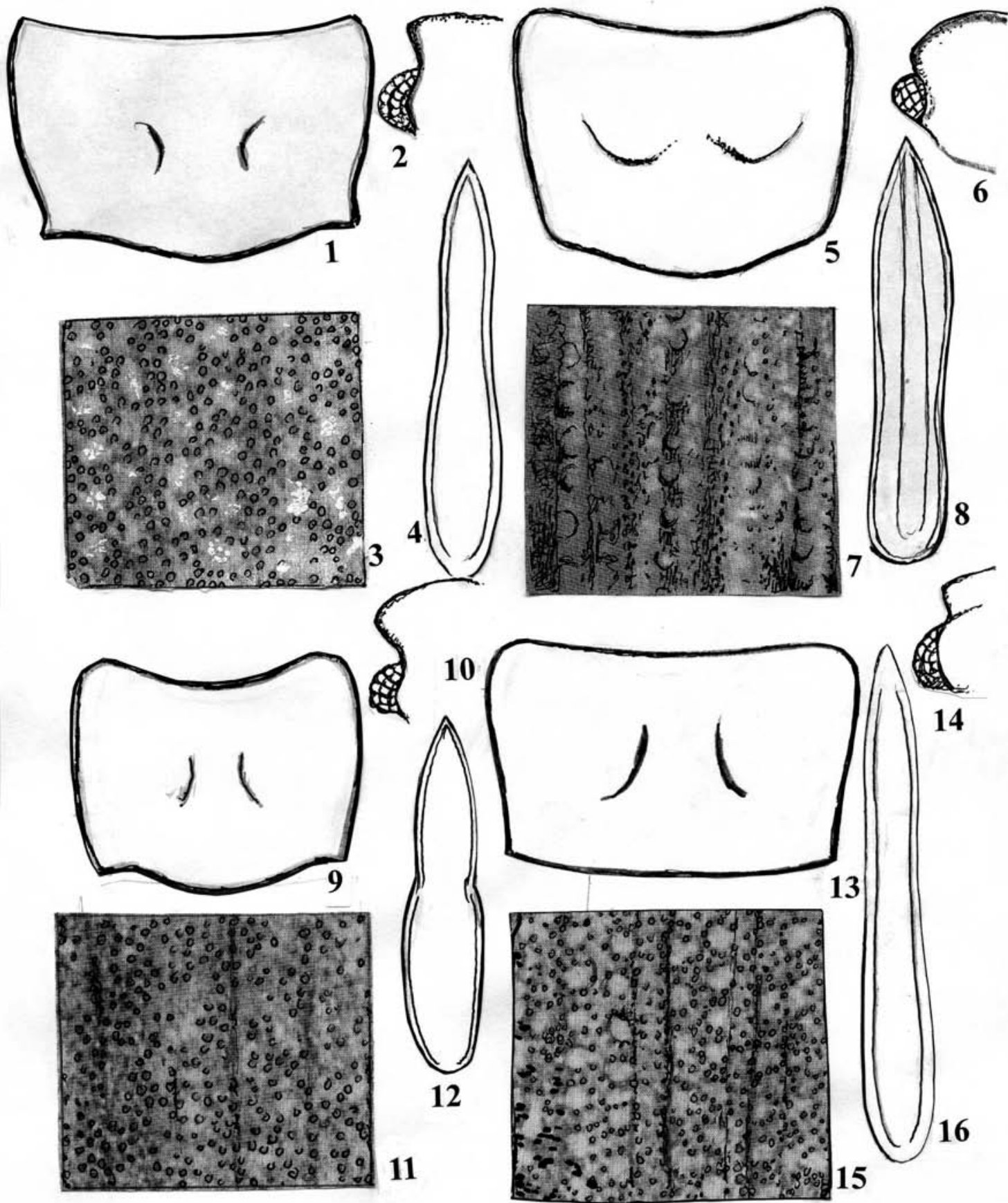

Figs. 1-16.- Cnemeplatia atropos stat. nov.: 1) Pronoto, ejemplar de Corfú (NRM); 2) Ojo; 3) Escultura elitral; 4) Edeago. Cnemeplatia rufa stat. rest.: 5) Pronoto (ejemplar de Tánger, HMNH); 6) Ojo; 7) Escultura elitral; 8) Edeago. Cnemeplatia theryi (Ejemplar de San Roque, CJF): 9) Pronoto 10) Ojo; 11) Escultura elitral; 12) Edeago. Cnemeplatia muchampsi Holotipo: 13) Pronoto; 14) Ojo; 15) Escultura elitral; 16) Edeago.

Figs. 1-16.- Cnemeplatia atropos stat. nov.: 1) Pronotum, specimen from Corfu (NRM); 2) Eye; 3) Elytral sculpture; 4) Aedeagus. Cnemeplatia rufa stat. rest.: 5) Pronotum, specimen from Tangiers (HMNH); 6) Eye; 7) Elytral sculpture; 8) Aedeagus. Cnemeplatia theryi (specimen from San Roque, CJF): 9) Pronotum; 10) Eye; 11) Elytral sculpture; 12) Aedeagus. Cnemeplatia muchampsiHolotype: 13) Pronotum; 14) Eye; 15) Elytral sculpture; 16) Aedeagus. 
Kaszab (1938) como la forma típica C. a. atropos. El carácter discriminatorio empleado por Español (1959), así como por Viñolas \& Cartagena, (2005) para separar Cnemeplatia atropos de C. rufa $(=C$. a. africana), es insuficiente, pues existen ejemplares en los que la sinuosidad posterior de los lados antes de la base ha desaparecido prácticamente (Fig. 17).

La figura 5 publicada por Kaszab (1938) representa un insecto con fosetas pronotales profundas, y con los élitros proporcionadamente bastante largos. La figura se adapta bien a la descripción original así como a la redescripción de Reitter (1904).

El tipo y las series de Nápoles del Museo de Basilea (NHB), corresponden a los ejemplares de Grecia y de Palencia que representamos (Figs. 27 y 28).

Cnemeplatia atropos descrita de Nápoles, fué redescrita como Autocera anticipes, por Wollaston (1857) sin localidad precisa, sospechando que el ejemplar único citado, debía provenir de Sicilia o de Egipto (por las circunstancias de su captura). Esta especie es citada por Reitter (1904) y por Porta (1934) de Italia. Gebien (1910) y Gebien (1939) la cita de Europa meridional, de la Península Ibérica, Noroeste de África, Egipto y Madeira. Cnemeplatia atropos es una especie que cohabita en Argelia con Cnemeplatia rufa, por lo que su distribución geográfica no se limita a la Península Ibérica, Italia, Sicilia, Hungría y Grecia, siendo nueva para Argelia y para Turquía. Baudi (1886) la cita de Chipre.

La cita de Koch (1935) de Egipto, basada en un ejemplar único de la colección Alfieri, hoy conservada en el Museo de Basel (col. Frey), y que hemos examinado, corresponde a una forma inédita que también existe en Sudán.

Lencina et al. (2007) citan C. atropos de la provincia de Murcia. Löbl \& Smetana (2008) citan de la fauna peninsular a $C$. atropos, donde ciertamente existe, mezclada con C. rufa, a pesar de haber sido excluìda por Viñolas \& Cartagena (2005), limitando erróneamente la distribución del género Cnemeplatia al extremo sur de la Península. El material colectado en España por Paganetti (NRM, NHMB), prueba que la distribución de Cnemeplatia atropos, llega por lo menos, hasta Palencia, alcanzando el NO de España y el sur de Portugal, confirmando los datos actuales de distribución según La Fuente (1935).

Cnemeplatia rufa Tournier, 1874 stat. rest.

Cnemeplatia rufa Tournier, 1874: 36.

Cnemeplatia atropos africana Kaszab, 1938:p. 79 fig. 6, p. 81, syn. nov.
Material eXAminado: Holotipo: Marruecos: Tánger: Tangiers, H. Tournier/Coll. M. Pic, Muséum national d'Histoire naturelle (MNHN), Paris.

Marruecos: Casablanca, Antoine leg. det. Z. Kaszab (3, NHMB); Tanger, Rolph (NHMB); Tánger, Escalera (2, MNCN); Argelia: Orán (NHMB); Argel, (sin más datos) Pérez Arcas, 364 1, MNCN); España: Málaga, Cobos y Mateu leg. (EEZA); Huelva: Parque Nacional Doñana, 24.VII. y 26.X. 2000, A.M. Cárdenas leg.; Cádiz, San Roque, 11.X.1992, col. Juan de Ferrer; Guardamar, Alicante, col. A. Castro Luque; Madrid, Aranjuez/ C. atropos Costa (F. Español det) (5, MNCN); Murcia: Jumilla, La Alquería, 29.XI.2003, J.L. Lencina leg. (suelo de olmo); Jumilla: El Portichuelo, 10.VIII.2005 y 1.XI.2006, suelo de olmo, J.L. Lencina; Charco de Ontur, 17.VII.2007, H.L. Lencina leg.; Albacete: Lietor; Puente de Hijar; Hellín, Río Segura, 2.XI.2003, suelo de olmo, J.L. Lencina leg. (todos en Col. Lencina); Palencia, Paganetti, (2, NRM); Portugal: Zeca, C. de Barros leg. (MNCN).

Se trata de una especie arbitrariamente sinonimizada por Gebien $(1910,1939)$ caracterizada por las proporciones del pronoto, más largo en relación al largo de los élitros, que en C. atropos, por los lados del mismo sin sinuosidad lateral prebasal y por el edeago (Figs. 5, 8, 20, 30).

Lencina et al. (2007), indican la necesidad de revisar el material ibérico atribuido a la subespecie C. atropos africana. Tras nuestro estudio podemos concluir que Cnemeplatia rufa, descrita de Tánger, es idéntica a C. atropos africana Kaszab (1938): Sintipo, Casablanca, 1933, Reitter/coll. A. Thery (HMNH, Budapest).

Español (1959, p. 62) describe el material estudiado por él, de Málaga, Cádiz y Murcia, comparado con material de Marruecos y de Túnez, confirmando con una cierta reserva, la presencia de Cnemeplatia atropos africana, en ciertas zonas de Andalucía y en Murcia. Evidentemente en el trabajo de Español (1959, p. 61 fig. 4 a.) representando Cnemeplatia atropos africana se produjo un error, remitiendo al lector a la fig. 4 a, e incurriendo en contradicción con la dicha figura, que corresponde a Cnemeplatia atropos atropos por la neta sinuosidad de los lados del pronoto en su tercio basal, y que es bien diferente de los contados ejemplares ibéricos estudiados por F. Español.

Los ejemplares del sur de Portugal y del sur de España y Marruecos, presentan el pronoto trapezoidal, poco transverso, nada cordiforme, los lados rectos, no sinuados en su tercio basal y los élitros aún más cortos (Figs. 5, 20). Caracteres que pueden así mismo comprobarse, comparando la figura 6 de Kaszab (1938), con la fotografía de los ejemplares típicos italianos (Aliquó et al., 2007). Sin embargo, sin examinar el material típico, ni la figura 6 original, ni la breve descripción de C. atropos africana 

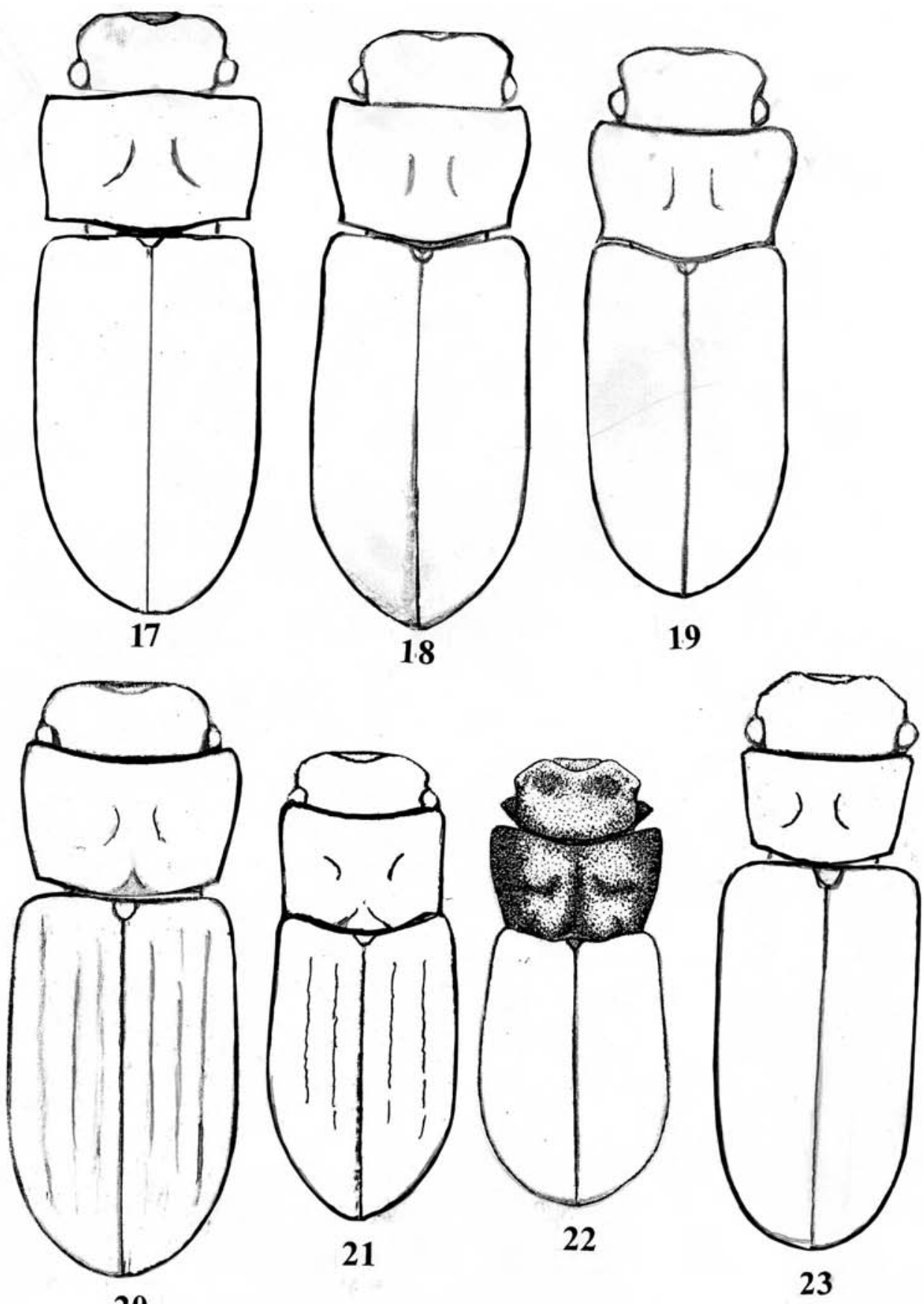

20

Figs. 17-23.- Figuras de Cnemeplatia: 17) C. atropos (Murcia); 18) C. atropos (Nápoles); 19) C. atropos (Grecia, Corfú); 20) C. atropos africana (Tipo, Casablanca); 21) C. theryi (Holotipo); 22) Figura original de C. theryi según Kaszab, 1938. 23) C. mouchampsi stat. nov.

Figs. 17-23. - Habitus of Cnemeplatia: 17) C. atropos (Murcia); 18) C. atropos (locotypical: Naples); 19) C. atropos (Greece, Corfu); 20) C. atropos africana (Type, Casablanca); 21) C. theryi (Holotype); 22) Original figure of C. theryi after Kaszab, 1938. 23) C. mouchampsi stat. nov. 
que dió Kaszab, son suficientes para la interpretación de estos insectos.

El catálogo de La Fuente (1935), cita Cnemeplatia atropos de Valencia (Moróder); Cádiz (López Cepero, Walker) y del sur de Portugal: Tavira (von Maltzan). Español (1959) refiere todas esta citas, que considera verosímiles, como correspondientes a la subespecie C. atropos africana. En este mismo trabajo, cita material procedente de Málaga (Cobos), Huércal Overa, Almería (F. Español) y de Murcia: Totana (coll. Balaguer), así como del Norte de África, de las localidades típicas de Marruecos: Casablanca (Antoine, Kocher), de Tánger (Escalera) y así mismo de Melilla, Granja Muluya (Pardo); de Nador (A. Cobos), que hemos examinado (EEZA). Estas últimas determinaciones de Marruecos, así como las de Cobos (in litt.): "alred. de Almería", 11.IX.1950, Mateu y Cobos leg. (EEZA) y de Málaga (24.VI.1946, Cobos Sánchez leg. EEZA), son confirmadas como correctas y por lo tanto corresponden a C. rufa.

Por el contrario, los ejemplares de determinados de la mano de F. Español (in litt.) en el MNCN como Cnemeplatia atropos de Aranjuez (5 ejemplares, etiquetas de M. M. Escalera), Madrid, también corresponden a $C$. rufa.

En conclusión: aparte de Marruecos, de donde fueron descritos los dos taxones sinónimos, la especie existe en Argelia y Túnez, así como en la Península Ibérica, donde convive con C. atropos.

Cnemeplatia mouchampsi Español, 1948 stat. nov. Cnemeplatia atropos mouchampsi Español, 1948: 316

MATERIAL EXAMINADO: Holotipo, macho, Jadotville, (actual Likasi), R. P. Congo, Museo de Ciencias Naturales de Barcelona.

Estudiamos este taxón, aunque descrito fuera del ámbito de este trabajo, y establecemos que las diferencias morfológicas observadas en las poblaciones estudiadas en la Península Ibérica y Norte de África con el ejemplar congoleño indican que se trata de especies diferentes.

Diferente de C. atropos y de C. rufa, por las proporciones del pronoto en relación con los élitros, que son así mismo proporcionalmente más anchos, separándose tambien de éstas por el largo mayor del mesosterno, tal como indica Español (1948) en su descripción. El edeago es tambien más largo que en estas especies. Sólo se conoce el holotipo. (Fig. 13-16, 23, 33)

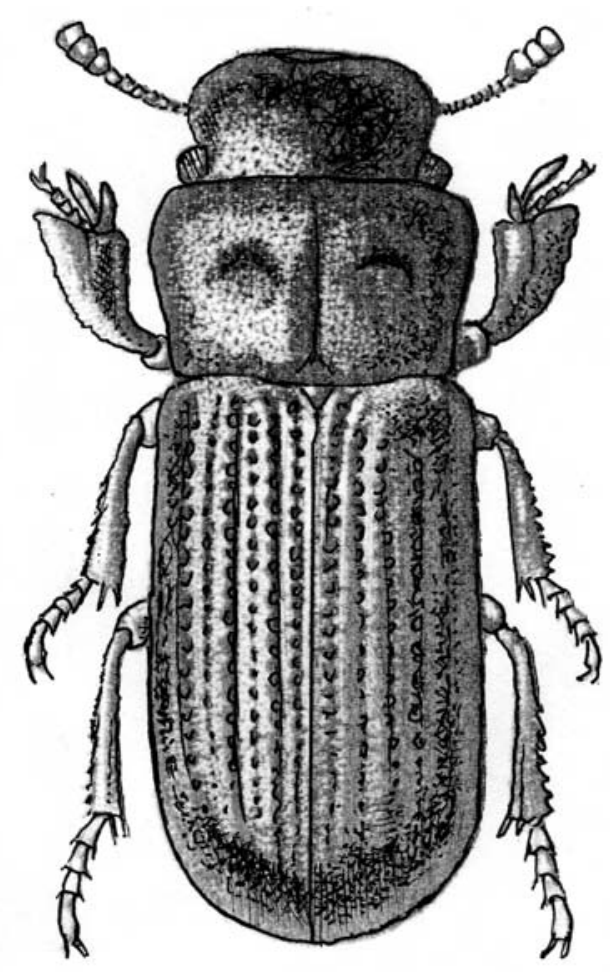

Fig. 24.- Ilustración original de Autocera laticeps = Cnemeplatia laticeps.

Fig. 24.- Autocera laticeps $=$ Cnemeplatia laticeps, original figure.

Cnemeplatia laticeps (Wollaston, 1857)

Autocera laticeps Wollaston, 1857: 155, fig. 2

Material eXaminado: Holotipo: Madeira, Sta Cruz to S. Antonio da Serra, 11.vi.1855/coll.Wollaston/Brit. Museum, The Natural History Museum, London. (sexo no examinado). Islas Canarias: Tenerife, F. Español det. (col. J. Ferrer), La Palma (col. Israelsson, NRM, Estocolmo).

Reconocible por sus ojos salientes, sub-hemisféricos, no cónicos, por las proporciones de los élitros, un poco más largos $(1.5 \mathrm{x})$ que en $C$. theryi $(1.4 \mathrm{x})$, de lados paralelos, sin la dilatación posterior característica del contorno antes del ápice, por el pronoto con fosetas y por la tercera costilla dorsal marcada (Figs. 24, 34).

La descripción, la figura original (Wollaston, 1857 , p. 155 fig. 2) (fig. 24) y el tipo, no corresponden bien a la figura y descripción de Kaszab, (1938, fig. 7). Una diferencia notable es la profundidad de las fosetas del pronoto del tipo, levísimas en la figura de Kaszab, (1938, fig. 7). Por otra parte, es de notar que el fuerte punteado de los éli- 

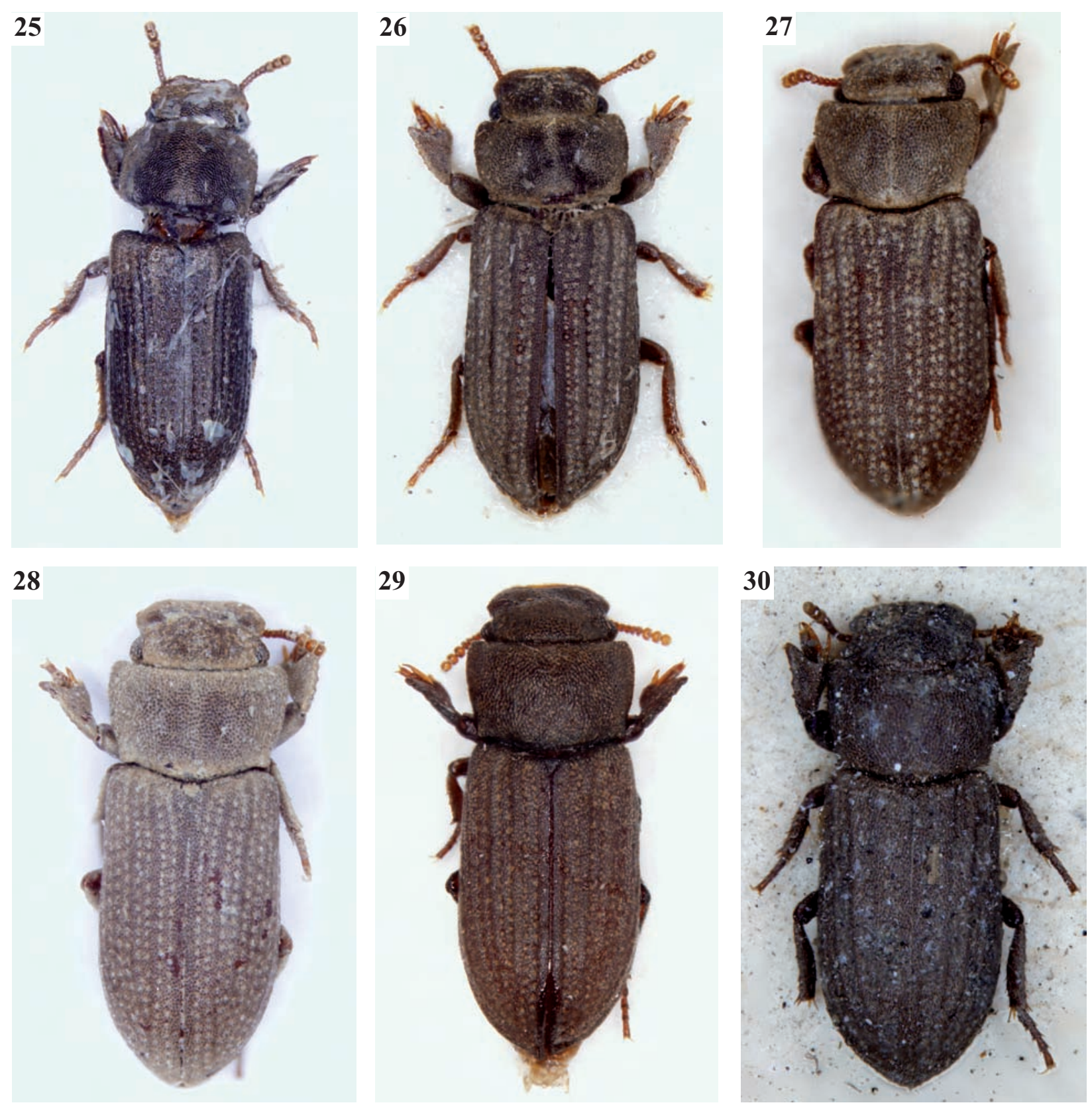

Figs. 25-30.- 25) Philhammus sericans de Coto Doñana. Imágenes de Cnemeplatia atropos stat. nov. de: 26) Turquía, 27) Corfú, 28) Palencia y 29) Murcia. 30) Cnemeplatia rufa stat. rest. de Casablanca.

Figs. 25-30. - 25) Philhammus sericans from Coto Doñana. Images of Cnemeplatia atropos stat. nov. from 26) Turkey, 27) Corfu, 28) Palencia and 29) from Murcia. 30) Cnemeplatia rufa stat. rest. from Casablanca. 

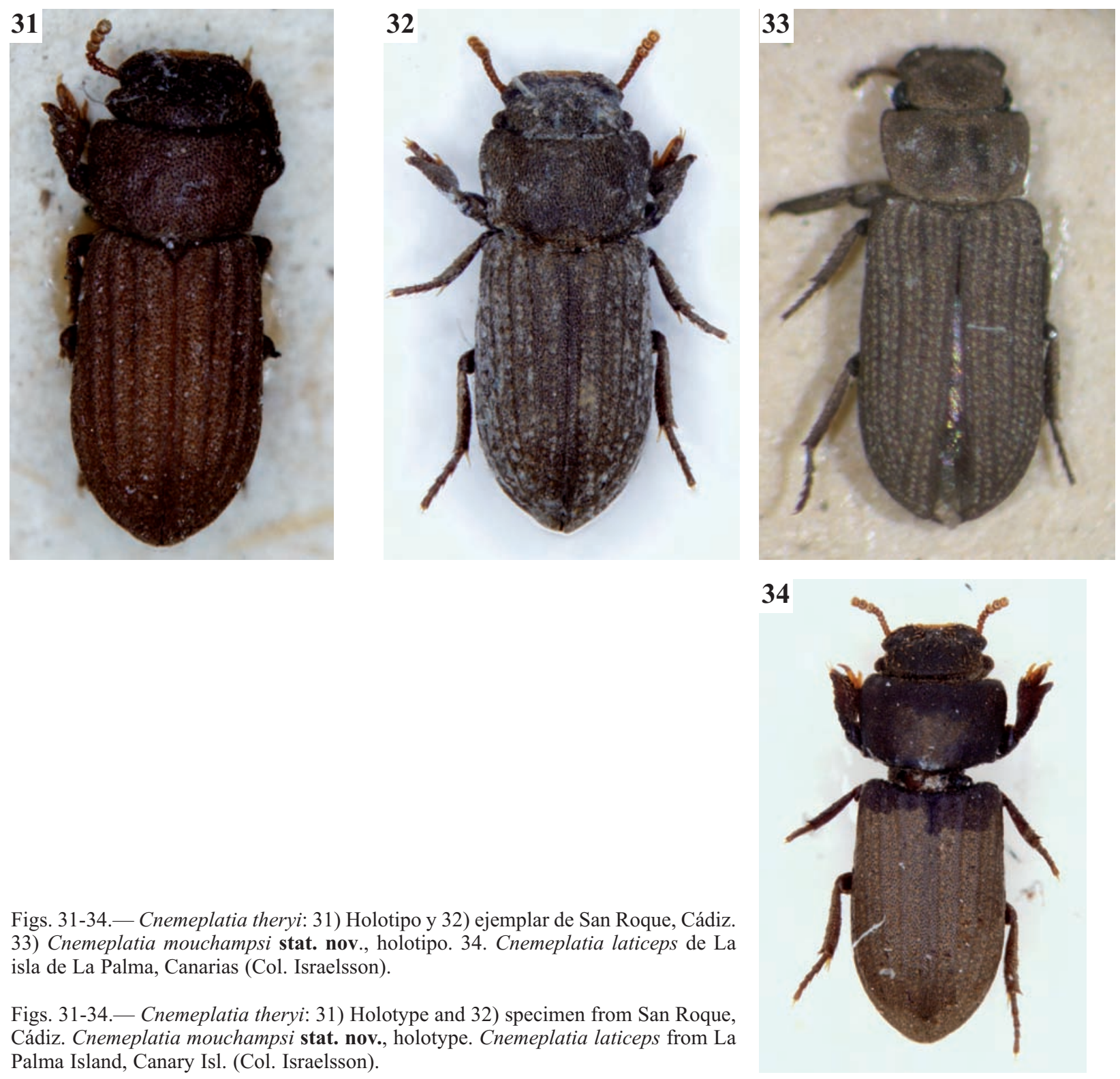

Figs. 31-34.-Cnemeplatia theryi: 31) Holotipo y 32) ejemplar de San Roque, Cádiz. 33) Cnemeplatia mouchampsi stat. nov., holotipo. 34. Cnemeplatia laticeps de La isla de La Palma, Canarias (Col. Israelsson).

Figs. 31-34.- Cnemeplatia theryi: 31) Holotype and 32) specimen from San Roque, Cádiz. Cnemeplatia mouchampsi stat. nov., holotype. Cnemeplatia laticeps from La Palma Island, Canary Isl. (Col. Israelsson).

tros en el ejemplar típico, es muy débil en los ejemplares, de $C$. laticeps de Tenerife, determinados por Español, desapareciendo por completo en los ejemplares de La Palma (figuras 24, 34).

La descripción original indica netamente las fosetas profundas del pronoto y el fuerte punteado elitral, caractéres típicos, son quizás propios y exclusivos de la población que habita la Isla de Madeira y que no corresponden exactamente a la figura de Kaszab (1938) que representa más bien las formas de las Islas Canarias. Un estudio en profundidad de las poblaciones que integran estas Cnemeplatia es indispensable.
Cnemeplatia theryi Kaszab, 1938

Cnemeplatia theryi Kaszab 1938

Cnemeplatia therzy (sic) Kaszab, Español, 1959

Material eXAminado: Holotipo: Sud Spanien. Algeciras, Simon leg. (Hungarian Museum of Natural History, Budapest). Cádiz, San Roque, Juan de Ferrer y J. L. Torres Méndez, 20.I.1973 (su colección y CJF), Chiclana, 17.I.2001 (bajo hojas de eucalipto); San Fernando, Cádiz, 21.XI.2007, P. Coello leg. (bajo hojas de acacia) (Col. ACT). No existen diferencias entre el holotipo y los ejemplares de Cádiz que hemos examinado.

Cnemeplatia theryi fue descrito sobre un ejemplar único de Algeciras, En la descripción original, el ojo se define como "kegelformig" (cónico), pero 
en una de las figuras presentadas por Kaszab (1938, p. 81, fig. 16), se representa un ojo casi redondeado, que no tiene correspondencia con la otra figura del habitus de esta especie, que presenta un ojo netamente cónico (fig. 14 cf. Kaszab, 1938, p. 79, fig. 16 y fig. 8). El tipo tiene los ojos subcónicos, no cónicos, es decir la mitad superior presenta una curva perfecta que a partir del medio se hace casi recta hacia atrás (fig. $21 \mathrm{cf} 22$ ).

La forma del holotipo no se ajusta totalmente, a la figura y a la descripción original, por presentar los ojos salientes, subcónicos, no cónicos, los húmeros marcados, aunque no tanto como laticeps, con cuatro costillas contando la sutural, no "tres", siendo todas enteras hasta los húmeros, los élitros subparalelos, no tan dilatados en su tercio apical, como en la figura original, por tener las fosetas pronotales y la depresión basal perfectamente marcadas, por presentar la tercera costilla lateral, completa, no "interrumpida antes de la zona humeral", como rezan las redescripciones de Español (1959) y Viñolas \& Cartagena (2005) de los ejemplares gaditanos. Estos autores publican una fotografía $413 \mathrm{C}$, que por el largo de los élitros, con respecto al pronoto y su forma, no se corresponde con el holotipo de $C$. theryi, ni con los ejemplares de esta especie examinados, si no que corresponde a C. rufa. Cnemeplatia theryi presenta los élitros más cortos y granulosos en relación al pronoto y la máxima anchura de los mismos en el medio, (ver fig. $21 \mathrm{y} 22$ ).

\section{Clave de las especies del género Cnemeplatia de Europa y NO de África}

1 Contorno lateral de los ojos saliente y semicircular (Figs

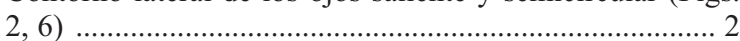

- Contorno lateral de los ojos saliente, subcónico (Fig. 10)

Pronoto subtrapezoidal y netamente transverso, 1.7 veces más ancho que largo y ligeramente subcordiforme, los lados del pronoto muy levemente sinuados a partir del tercio basal (fig. 1), de forma que éstos son rectos y ligeramente salientes hacia atrás. Elitros proporcionalmente largos en relación al pronoto, 3 veces más largos que éste (figs. 17, 18, 19), su anchura depasa la anchura máxima del pronoto. Estrías elitrales bien aparentes, sin vestigios de costillas

C. atropos

- Pronoto trapezoidal y poco transverso, 1.3 más ancho que largo, con los lados completamente rectos, del borde anterior a la base, no sinuados en su tercio basal (fig. 5). Elitros proporcionalmente más cortos en relación al pronoto, menos de 3 veces más largos que éste y con costillas vestigiales, perceptibles lateralmente, los puntos apenas visibles (figs. 5, 20) C. rufa
3 Pronoto netamente transverso, casi dos veces más ancho que largo, su máxima anchura en el medio. Elitros proporcionalmente largos en relación al pronoto, siendo dos veces y media más largo que éste y una vez y media más largos que anchos, con costillas leves pero bien perceptibles dispuestas alternativamente (fig. 24)

C. laticeps

- Pronoto poco transverso, una vez y media tan largo como ancho, su máxima anchura en el tercio anterior (fig. 9). Elitros proporcionalmente más cortos, 2.5 veces más largos que el pronoto, una vez y media más largos que anchos, con costulación perceptible, pero lateral (figs. 31, 32)

C. theryi

\section{AGRADECIMIENTOS}

In memorian a Zoltán Kaszab y a Francisco Español y Juan de Ferrer, que en su día, comunicaron generosamente sus materiales a JF.

Este estudio ha sido posible gracias al apoyo económico a JF de COLPARSYS, Muséum national d'Histoire naturelle, Paris, 2002 y de SYS-RESOURS, para visitar The Natural History Museum, Londres, 2003. Este trabajo ha recibido así mismo apoyo de Synthesys Project PL TAF 5654 and PL TAF 882 (http://www.synthesys.info/), financiado por el Programa de Investigación científica de la Comunidad Europea, para el estudio de las infraestructuras, FP6 "Estructuración del Programa de Investigación Europea (European Community Research Infrastructure Action under the FP6 "Structuring the European Research Area Programme"). Por su cordial acogida durante el estudio de las colecciones que custodian, se agradece a MM. Max Barclay; The Natural History Museum, a Claude Girard, Muséum national d'Histoire naturelle, París, así como a Eva Sprecher, del Naturhistorisches Museum, de Basilea, a Otto Merkl, Termeszéttudomanyi Muzéum, Budapest, por el envío de los tipos de Zoltán Kaszab, imprescindibles para el estudio del género; A Isabel Izquierdo y Mercedes Paris García, conservadoras del Museo Nacional de Ciencias Naturales, Madrid, a Glória Masó, del Museo de Ciencias Naturales de Barcelona, a Jesús Benzal, de la Estación Experimental de Zonas Áridas (EEZA), Almería, en fin a Fredrik Ronquist, Kjell Arne Johanson, Kevin Holston, Bert Gustafsson, Bert Viklund y Niklas Jönsson, del Naturhistoriska riksmuseet, Estocolmo, a J. L. Torres Méndez, de Algeciras y a José Luis Bujalance, Baena, por envío de material.

\section{Referencias}

Aliquò, V., Rastelli, M., Rastelli, S. \& Soldati, F., 2007. Piccole Faune. Coleotteri Tenebrionidi d'Italia. Darkling Beetles of Italy. CD-Rom. Museo Civico di Storia Naturale Carmagnola (TO), Associazione Naturalistica Piamontese (ANP), Progetto Biodiversità. Comitato Parchi-Centro Studi (Roma). ISBN 88-901201-8-5 IM2-07

ArdoIN, P., 1976. Nouvelles espèces africaines de Tenebrionidae. COL. Bulletin de la Société entomologique de France, 81: 148-152.

Baudi e Salve, F., 1886. Coleotteri tenebrionidi delle Collezione Italiane. Tribu 26. Opatridi. Bolletino della Societá Entomologica Italiana, 7: 209-237. 
Bouchard, P., Lawrence, J. F., Davies, A. E. \& Newton, A. F., 2005. Synoptic Classification of Worl Tenebrionidae (Insecta, Coleoptera) with a review of Family-group names. Annales Zoologici, Warszawa, 55(4): 499-530.

Cárdenas, A. M., Bujalance, J. L., Hidalgo, J. M. \& FERrer, J., 2004. First data on the presence of the Philhammus Fairmaire 1870 (Col. Tenebrionidae) genus in Europe. Nouvelle Revue d'Entomologie, (N.S.), 21(3): 211-212.

CostA, A., 1847. Descrizione di alcuni Coleotteri del regno di Napoli. Annali dell'Accademia degli aspiranti Naturalisti. Napoli, (2), 1: 134-162.

CsIKI, E., 1953. Über neue und bekannte Coleoptera aus Ungern und angränzande länder. Annales Historico Naturales Musei Nationalis Hungarici (N.S.), 3: 115135.

EsPAÑOL, F., 1948. Notas sobre Coleópteros Africanos: 2. El género Cnemeplatia (Tenebrionidae) en el Congo Belga. Eos, 24: 315-318.

ESPAÑOL, F., 1959. Datos para el conocimiento de los Tenebriónidos del Mediterráneo Occidental, XVI. Los Melanimini de la Península Ibérica y Marruecos. Graellsia, 17: 59-70.

EsPAÑOL, F., 1962. Los Opatrinae de las Islas Canarias. Col. Tenebrionidae. Eos, 38(1): 201-221.

FAIRMAIRE, L., 1870. Essai sur les Coléoptères de Barbarie. $7^{\text {me }}$ partie. Annales de la Société entomologique de France, $4^{\mathrm{ime}}$ Série, 10: 392-404.

FAtTorini, S., 2005. Fauna Europae. In: P. Audidio (ed.). Fauna Europaea. Coleoptera 2, versión 1.2 http//:www. faunaeur.org

Ferrer, J., 1995. Philhammus brincki n. sp. from South Africa (Coleoptera, Tenebrionidae). Nouvelle Revue d'Entomologie, N.S., 12(2-3): 145-148.

Ferrer, J., 2006. Revisión crítica del libro Fauna de Tenebrionidae de la Península Ibérica y Baleares. Vol. 1, Lagriinae y Pimelinae. VIÑOLAS y CARTAGENA, 2005. Boletín de la Sociedad Entomológica Aragonesa, 39: 463-466.

Ferrer, J. \& OdegAard, F., 2005, Darkling beetles from Central America, with systematic notes (Coleoptera, Tenebrionidae). Annales Zoologici, Warszawa, 55(4): $437-465$.

Fuente y Morales, J. M. DE LA, 1935. Catálogo sistemático-geográfico de los Coleópteros observados en la península ibérica, Pirineos propiamente dichos y Baleares (continuación). Boletín de la Sociedad entomológica Española. Fam. Tenebrionidae. Vol. 16. (1933): 96-111; Vol. 17 (1934): 17-32; Vol. 18 (1935): 17-32. (Tenebrionidae: Catálogo pp.: 23-111)

Gebien, H., 1910. Katalog der Tenebrioniden (1-3). In: W. Junk und S. Schinkel (eds.). Coleopterorum Catalogus, Pars 15 \& 22. W. Junk. Berlin. 740 pp.
GebIEN, H., 1939. Katalog der Tenebrioniden, Teil 2, Mitteilungen der Münchner Entomologische Sällschaft, 28(3): 445-764.

JACQUELIN DU VAL, C., 1861. Manuel Entomologique. Genera des Coleóptères. Comprenant leur classification en familles naturelles, la description de tous les genres, des tableaux synoptiques destinés à faciliter leur étude, le Catalogue de toutes les espèces, de nombreux dessins au trait de charactéres et plus de treize cents types representant un ou plusieurs insectes de chaque genre, dessinés et peints d'aprés nature avec le plus grand soin par M. Jules Migneaux. Tome troisième. A. Deyrolle. Paris. 464 pp, 100 pl.

Kaszab, Z., 1938. Die Arten der Gattung Cnemeplatia Costa und Psilachnopus Reitter. Entomologiska Tidskrift, 59(1-2): 77-82.

KaszaB, Z., 1966. Die Arten der Gattung Lepidocnemeplatia. Entomologische Arbeiten aus dem Museum G. Frey, 17: 39-47.

KocH, C., 1935 Wissenschaftliche Ergebnisse der Entomologischen Expedition seinen durchlaucht des Fuersten A, Della Torre e Tasso nach Aegypten und auf die Halbinsel Sinai. Bulletin de la Société Royale Entomologique d'Egypte, 19: 2-111.

Lencina Gutiérrez, J. L., Ortiz Cervantes, S. \& Rubio LozAno, R. M., 2007. Nuevas citas de Tenebrionidae (Coleoptera) del Sureste de la Península Ibérica. Boletín de la Asociación española de Entomología, 31(1-2): 185-192.

LÖBl, I. \& SMetanA, A., 2008. Catalogue of Palaearctic Coleoptera, vol. 5, Tenebrionoidea. Apollo Books. Stenstrup. $670 \mathrm{pp}$.

Medvedev, G. S., 1982. Novyy vid zhukov-chernotelok roda Cnemeplatia Costa (Coloptera, Tenebrionidae) iz Karakalpakskoy, ASSR. Trudy Zoologischeskogo Instituta Akademii Nauk, SSSR, 110: 40-41.

Medvedev, G. S., 1991. Novyy chernotelki trib Stenosini i Cnemeplatiini (Coloptera, Tenebrionidae) mirovoy fauni. Entomologischeskoe Obozrenie, 70: 557-570.

Medvedev, G. S., 2005. New species of Tenebrionid beetles from Botswana and Afghanistan. (Coleoptera, Tenebrionidae). Annales Zoologici, Warszawa, 55(4): 561-563.

PorTA, A., 1934. Fauna Coleopterorum Italiana. Vol. 4. Heteromera. Stabilimento Tipografico Piacentino. Piacenza. $415 \mathrm{pp}$.

REICHARDT, A., 1936. [Darling beetles of the Tribu Opatrini of the Palaearctic Region (Coleoptera, Tenebrionidae).] Keys to the Fauna of the U.R.S.S., 19: 1-224 (en ruso).

ReITTER, E., 1904. Bestimmungs-Tabellen der Tenebrioniden-Unterfamilien Lachnogyini, Akidini, Pedinini, Opatrini und Trachyscelini, aus der Europa und angrenzenden Ländern. Verhandlungen des naturforschenden Verein in Brünn, 39 : 25-289. 
Serrano, J., Lencina, J. L. \& AndúJar, A., 2003. Distributions patterns of Iberian Carabidae (Insecta, Coleoptera). Graellsia, 59(2-3): 129-153.

TOURNIER, H., 1874. Diagnoses de quelques Coléoptères européennes et circumeuropéens. Annales de la Société Entomologique de Belgique, 17(ComptesRendus des Séances): lxxxvi-lxxxix.

TRIPLEHORN, C., 1987. A new species of Lepidocnemeplatia, from Brazil, with distributioal notes on other species in the genus (Coleoptera, Tenebrionidae). The Coleopterist's Bulletin, 41: 335-338.

Wollaston, T. V., 1857. Catalogue of the Coleopterous Insects of Madeira in the collection of British Museum. Taylor \& Francis. London. $43+634$ pp., 1 pl.

VianA, M. J., 1960. Una nueva especie del género Lepidocnemeplatia Kaszab de la Rep. Argentina. Actas del Primer Congreso Sudamericano de Zoología, La Plata: 259-265.
Viñolas, A. \& Cartagena, M. C., 2005. Fauna de Tenebrionidae de la Península Ibérica y Baleares. Vol. 1, Lagriinae y Pimeliinae. Argania Editio. Barcelona. 428 pp.
Recibido, 16-VII-2008

Aceptado, 13-XI-2009 Publicado, 31-XII-2009 